\title{
iERBMF
}

\section{A study on the critical barriers to sustainable manufacturing in SMEs}

\author{
An-Yuan Chang ${ }^{\text {a1 }}$ \\ aDepartment of Industrial Management, National Formosa University No. 64, Wun Hwa \\ Road, Hu Wei, Yunlin632, Taiwan
}

\begin{abstract}
Sustainable manufacturing implies the creation of products that utilize minimum resources, has minimum negative impacts on environment and are safe for society at an affordable cost. In the literature of sustainability studies it has been confirmed that there exist significant and positive relationships between corporate social responsiveness and profitability. The improvement of sustainability management and performance for a company is an opportunity to firm's development and growth rather than a threat. SMEs should effectively utilize their limited resources and take into consideration the differences in elaborating their sustainable development. This research is focused on the study of drivers and barriers to sustainable manufacturing SMEs in Taiwan. An attempt is tried to consider the characteristics of Taiwanese SMEs and integrate a mixed multiple attribute decision making model for identifying the key factors of barriers to sustainable manufacturing SMEs. An attempt is tried to consider the characteristics of Taiwanese SMEs and integrate a mixed multiple attribute decision-making model for identifying the key factors of barriers to sustainable manufacturing SMEs. The results show which barriers are in the most critical position. The details of how to overcome the barriers and what efforts on improving those drivers will provide important decision making for business and government decision-makers. In this study the application of fuzzy Delphi method (FDM) is used to screen barriers and drivers, and the interpretive structure modeling (ISM) will be further used to build a hierarchical relationship between the barriers to sustainable manufacturing.
\end{abstract}

Keywords: Sustainable manufacturing, SMEs, barrier, FDM, ISM 